\title{
Shelf life of rechargeable laryngoscope batteries without recharging
}

\author{
Andrew D. Milne, MD • Paul A. Brousseau, BEd
}

Received: 2 May 2012/Accepted: 15 May 2012/Published online: 30 May 2012

(c) Canadian Anesthesiologists' Society 2012

\section{To the Editor,}

Proper laryngeal illumination is a crucial component of providing optimal conditions for tracheal intubation under direct laryngoscopy. Intubating equipment is often used and checked on a daily basis in the operating room; however, laryngoscopes often lie inactive for prolonged periods in emergency airway kits for the general wards, the emergency room, critical care units, or out-of-hospital use. Limited data are published on the life span of laryngoscope batteries or the safe storage period for emergency airway kits. We undertook to evaluate the useful "shelf life" of a commonly used laryngoscope device without battery recharging between uses.

Institutional research ethics board approval was unnecessary for this simple bench test of a single laryngoscope to evaluate battery life between full charges. A new Heine 3.5 volt Xenon laryngoscope handle with a new nickel metal hydride (NiMH) battery and a new Heine Macintosh \#3 Classic reusable fibreoptic stainless steel blade (Herrsching, Germany) was evaluated. The rechargeable battery was fully charged at the start of this study. The light intensity delivered from the handle and blade unit was tested daily using a previously described testing chamber and light meter. ${ }^{1}$ Light output was recorded after $30 \mathrm{sec}$ of usage on each day (Figure). Testing was stopped on day 181 after the light output had dropped to 2,000 lux.

This bench-top test shows that new NiMH battery sets can hold their charge for prolonged periods of time without

A. D. Milne, MD $(\varangle) \cdot$ P. A. Brousseau, BEd

Dalhousie University, Halifax, NS, Canada

e-mail: admilne@dal.ca requiring recharging. Testing was stopped arbitrarily once the light output had reached 2,000 lux as this is the threshold level that our institution had deemed as a minimum safe light requirement prior to introduction of the new International Organization for Standardization (ISO) guidelines of 500 lux. $^{2}$ Our observations are limited by the fact that we tested a single new device with only one type of rechargeable battery (i.e., NiMH vs lithium or nickel cadmium batteries), and we did not examine battery "memory" effects. Despite these limitations, these observations over 181 days do help to characterize performance of these new laryngoscopes over prolonged storage periods under minimal usage conditions. These data should be useful to other institutions for equipment life cycle management and planning and for establishing quality control testing protocols for emergency airway equipment.

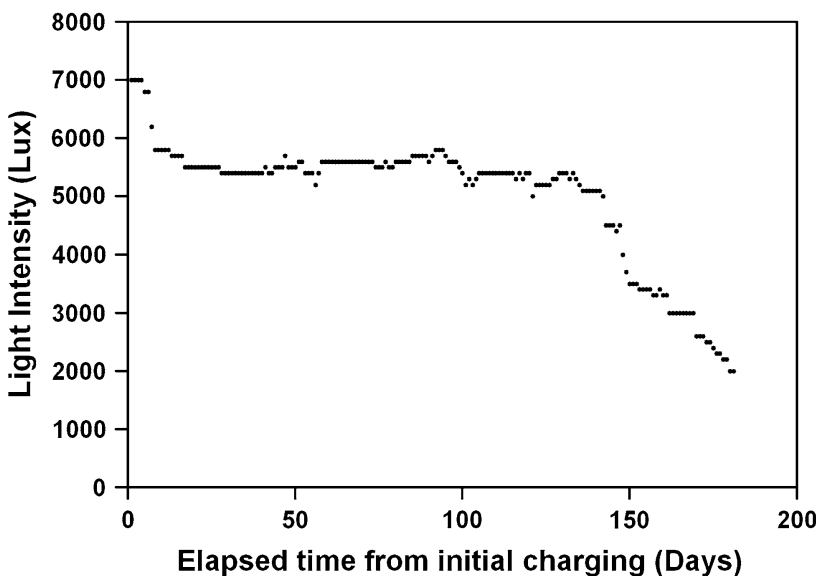

Figure Effect of daily usage on light output. Data shown are for a new rechargeable laryngoscope used for $30 \mathrm{sec}$ each day without recharging between uses 
Funding This study was unfunded.

Conflicts of interest None declared.

\section{References}

1. Cheung KW, Kovacs G, Law JA, Brousseau P, Hill W. Illumination of bulb-on-blade laryngoscopes in the out-of-hospital setting. Acad Emerg Med 2007; 14: 496-9.
2. International Organization for Standardization. Anaesthetic and respiratory equipment - laryngoscope for tracheal intubation. Geneva, Switzerland. ISO 7376:2009. Available from URL: http:// www.iso.org/iso/catalogue_detail.htm?csnumber=44073 (accessed May 2012). 